\title{
DOSSIER
}

España y la I Guerra Mundial

Cien años después

\section{España y Francia en la Primera Guerra Mundial}

Spain and France in First World War

\author{
Jean-Marc Delaunay \\ Universidad París 3 Sorbona Nueva \\ jmdelaun@univ-paris3.fr
}

Cómo citar este artículo/Citation:

Jean-Marc DELAUNAY (2017), “España y Francia en la Primera Guerra Mundial”, Hispania Nova, 15, págs. 303-315, DOI: https://doi.org/10.20318/hn.2017.3491

Copyright: (c) HISPANIA NOVA es una revista debidamente registrada, con ISSN 1138-7319 y Depósito Legal M 9472-1998. Los textos publicados en esta revista están -si no se indica lo contrario- bajo una licencia Reconocimiento-Sin obras derivadas 3.0 España de Creative Commons. Puede copiarlos, distribuirlos y comunicarlos públicamente siempre que cite su autor y la revista y la institución que los publica y no haga con ellos obras derivadas. La licencia completa se puede consultar en: http://creativecommons.org/licenses/by-nd/3.0/es/deed.es
\end{abstract}

Resumen: En el marco de la segunda gran fase denominada euroafricana, de la relación hispanofrancesa desde la caida de Napoleón I- situada en la Primera Guerra Mundial, no cambiaron sustancialmente sus fundamentos desde los años anteriores, es decir desde los acuerdos coloniales de 1900-1904. El conflicto transformó ciertos elementos relativamente secundarios como la caida de la deuda exterior española en manos francesas o la presencia económica francesa al sur de los Pirineos, en el marco de una debilitación general de Francia. Las grandes cuestiones fronterizas, migratorias, comerciales y africanas de antes de guerra permanecieron intactas entre los dos paises. La Segunda Guerra Mundial tampoco cambiará las bases principales de esta relación tan antigua como amplia. Habrá que esperar la descolonización de los años cincuenta-sesenta para observar una evolución notable que llegará hasta nuestros dias.

Palabras clave: relaciones hispano-francesas, Primera Guerra Mundial, neutralidad, relaciones internacionales.
Abstract: As part of the second great stage of the French-Spanish relationship since the fall of Napoleon, called Eurafrican, the WW1 - considered like European by the Spaniards - don't change the foundations established by the colonial agreements of 1900-1904. These Great War only transformed components relatively secondaries, like the lost by the French bondholders of a very large part of the external Spanish debt or the decline of the French activities in the Spanish economic world who can be considered like a small part of the general decadence of France after the terrible suffered War. The great deals were intacted, in the matters of borders, migrations, trade and in Africa. The two countries, the belligerent and the neutral, resolved wiht difficulties these contentious in the twenties. No more, the WW2 will change these relationship until the decolonization in the fifties-sixties of the last century.

Keywords: international relations, First World War, neutrality, Spanish-French relations 


\section{ESPAÑA Y FRANCIA EN LA PRIMERA GUERRA MUNDIAL}

Il s'agit ici d'un essai et non d'un article classique. Le but n'est pas de narrer et d'analyser l'évolution des relations franco-espagnoles durant la Grande Guerre et ses conséquences d'aprèsguerre - qui fera l'objet d'une publication assez prochaine ${ }^{1}$ - mais d'esquisser une perspective visant à démontrer que le conflit n'a pas altéré en profondeur les liens bilatéraux entre les deux pays même s'il en a inévitablement modifié certaines données particulières.

Cette affirmation préliminaire est à intégrer dans le temps long, depuis le grand bouleversement de 1813-1815 qui, lui, a affecté la relation par la défaite de l'un contre l'autre, symbolisée par la retraite des Français de la péninsule ibérique devant une coalition constituée d’Espagnols puissamment aidés par les Britanniques - maîtres de la manœuvre - et les Portugais. Du traité de Valençay (1813) - à nos jours (2016), cette relation franco-espagnole a connu globalement trois phases.

Si la première fut encore mondiale (au XIX siècle), la seconde étape des relations francoespagnoles - qui court des années 1900 au début des années 1960 - est essentiellement bicontinentale, eurafricaine. C'est donc dans ce contexte à durée alors indéterminée que s'inscrit la Première Guerre mondiale. Certes, la conquête de l'Algérie dans les années 1830 avait lancé un voisinage par le rôle des Baléares sur la route entre la métropole française et sa nouvelle possession nord-africaine (Marseille/Port-Vendres vers Alger/Oran), mais surtout par une forte immigration ibérique - précoce - à l'ombre du glaive français. De même, la concurrence aux frontières de ce territoire français et des vieilles enclaves espagnoles (Melilla notamment), marquée par l'occupation du médiocre - mais stratégique - archipel des Chaffarines (1847/1848), devait prendre l'aspect d'une approche progressive des espaces de souveraineté de ces deux puissances à périmètre encore mondial, l'une sur le mode offensif tous azimuts, l'autre sur le mode défensif d'une retraite générale. Finalement, à la fin du siècle, avec la disparition - par défaite militaire ou par rachat - de ses possessions américaines et océaniennes, l'Espagne perdait sa taille mondiale, rebondissant alors vers l'Afrique, au détriment de son ancrage européen. Son principal concurrent reconnu depuis les conférences de Berlin de 1884/1885 et de Bruxelles de 1890 - en Afrique équatoriale et au Sahara occidental - mais aussi plus discrètement au Maroc (conférence de Madrid 1880), c'était la République française qui, malgré les coups d’arrêt de Fachoda et d'Oman, demeurait une puissance qui constitue alors son second empire colonial, derrière la Grande-Bretagne rivale. La politique anti-allemande de Delcassé, ministre des Affaires étrangères de 1898 à 1905 - s'appuya sur une réaffirmation de l'alliance franco-russe, l'établissement d'une nouvelle

\footnotetext{
1 Jean-Marc Delaunay, Guerres d'Europe et d'Afrique. Les relations franco-espagnoles d'août 1914 à la fin des années 1920. Ce travail s'appuiera sur une importante documentation française, espagnole, allemande et anglaise accumulée des années 1980 à 2000 (ainsi que sur des documents diplomatiques italiens et nord-américains) sans oublier une bibliographie - jamais exhaustive - et une production universitaire inédite, jamais totalement recensée ou accessible.
} 
entente franco-anglaise, la neutralisation de l'hostilité italienne mais aussi sur l'entente francoespagnole ignorée des historiens les plus chevronnés. Après une tentative ratée d'entente secrète en novembre 1902 qui échoua par le refus final des Français de signer un accord qui eût donné Fez à I'Espagne, une entente coloniale - avec l'accord britannique - fut paraphée en octobre 1904, qui devait favoriser la conclusion d'autres accords frontaliers et ferroviaires sur le seul point de contact multiséculaire des deux pays, la frontière pyrénéenne. À cette mitoyenneté européenne allaient se joindre ainsi - pour une durée effective indéterminée (de trente à soixante ans selon les secteurs) bien d'autres voisinages africains de souveraineté (Sahara occidental/Mauritanie, Rio Muni/Gabon ${ }^{2}$ ) ou de protectorat (Maroc) aujourd'hui disparus. Les accords de 1900 (publics) sur les premiers territoires cités d'Afrique saharienne et équatoriale et l'accord (secret 1904) et le traité (public 1912) sur le Maroc en furent les bases encore visibles de nos jours par les tracés frontaliers alors fixés, notamment pour les espaces cités au sud de l'oued Draa. À signaler que deux ans et demi après Fachoda, les Français évacuèrent leurs garnisons du Rio Muni sur l'Équateur, dans la nuit du 20 au 21 juillet 1901, au profit de forces espagnoles mais c'était par un accord diplomatique préalable (accord du 28 juin 1900) et non par une confrontation de terrain comme face aux forces anglo-égyptiennes. En août 1914, Paris et Madrid étaient engagés dans une pacification lourde - initialement séparée - au Maroc qui se poursuivra plus au sud jusqu'au cœur du XX ${ }^{e}$ siècle (confins du Sud-Maroc et du Sahara occidental en 1933-1934), complices distants dans le cadre d'un "impérialisme de meute" qui a caractérisé le partage final de l'Afrique entre les années 1880 et 1920.

Alors que l'on évoqua même une perspective d'alliance en 1913, après la conclusion du traité sur le partage du Maroc signé à Madrid en novembre 1912, l'Espagne ne parut jamais être en position d'être un partenaire solide ${ }^{3}$. La parole du roi sur la tranquillité pyrénéenne en 1905 et 1913 fut bien sûr recueillie et notée avec intérêt. Lui vivant, jamais son pays ne menacerait la France dans son dos. Une Espagne résolument démocratique et occidentale ? Pourtant, après la guerre, dans une lettre du 10 mai 1919, l'universitaire Miguel de Unamuno devait confier à un correspondant français, Maurice Legendre, que c'était la germanisme qui avait gagné la partie idéologique en Espagne malgré la victoire des alliées et associés ${ }^{4}$. Un germanisme lié à la défense d'une neutralité espagnole intangible permettant notamment de maintenir des agents ou des réfugiés ennemis de la France sur place en cas de guerre générale. En stratégie, on tourne toujours le dos à quelqu'un.

\section{UNE NEUTRALITE INEVITABLE?}

La neutralité a été l'objet d'études et d'analyses anciennes mais la belligérance encore davantage, qui a pu apparaître comme un état naturel des relations inter-tribales, inter-ethniques, inter-dynastiques, inter-religieuses puis inter-nationales. S'affirmer en s'opposant paraît plus naturel

\footnotetext{
${ }^{2}$ Voir le vol. 2 (Relations coloniales) de l'ouvrage évoqué note 10, p. 119

3 J.-M. Delaunay, "L'Espagne, un allié oublié ? Les relations franco-espagnoles au début du XX $\mathrm{X}^{\mathrm{e}}$ siècle", Relations Internationales, $\mathrm{n}^{\circ} 106$, été 2001, p. 151-163.

4 J.-M. Delaunay, "Souvenir de Miguel de Unamuno, 1936-1986. Ses liens avec Maurice Legendre et la Casa de Velázquez", Mélanges de la Casa de Velázquez, Madrid, 1986 (tome XXII), p. 401-418. Les États-Unis étaient depuis 1917 une puissance associée et non alliée.
} 
que de s'abstenir de participer qui exige déjà un niveau de réflexion supérieur à celui de l'engagement violent ${ }^{5}$.

Jeune étudiant d'histoire en Sorbonne, à la sortie de la crise de mai 1968, j'avais longtemps considéré la Première Guerre mondiale comme initiatrice d'une nouvelle époque de l'humanité par sa longueur, par sa violence, par son étendue et par ses conséquences. L'aube du XX $X^{\mathrm{e}}$ siècle en somme. Dix ans plus tard, à la demande du directeur de thèse que j'avais choisi - Jean-Baptiste Duroselle, Professeur $\mathrm{d}^{\prime} H$ istoire des relations internationales ${ }^{6}$ - je me mis à travailler sur les relations franco-espagnoles. Pour des raisons linguistiques et familiales, j'avais initialement voulu me pencher sur les liens francoallemands dans le cadre des bornes chronologiques alors permises par l'ouverture des archives diplomatiques françaises (la fin des années 1920) mais les contraintes et avantages académiques et scientifiques du moment me firent accepter ce défi espagnol ${ }^{7}$. La tranche d'étude fut alors fixée sur la période 1898-1929 (voire 1931), soit autour de la Première Guerre mondiale. Dans cette large perspective, la capacité de chevaucher ce traumatisme européen, voire mondial, m'avait paru intéressante car la plupart des thèses s'arrêtaient alors à la guerre de 1914, l'étude de la guerre ellemême étant déjà considérée comme le travail d'une autre époque avec d'autres thèmes comme je l'ai souligné d'entrée. Pourtant, la maigreur des études relatives aux relations franco-espagnoles largement ignorées de tous après 1815 et surtout après $1870^{\circ}$ - notamment à propos du Maroc - m'obligea à limiter l'ampleur de ma thèse d'État à l'avant-conflit car il y avait trop à dire avant d'arriver à l'été 1914. C'est mon plus grand regret. Dès les années 1980, j'avais espéré démontrer que la dite guerre européenne générale (ou presque) n'avait pas changé grand chose à la relation fixée dans les années 1900-1904. Qu'il y avait en quelque sorte la circonstance et la constance, la rupture et la continuité. Pour la thèse d'État, je n'ai donc pas pu échapper à cette coupure de l'été 1914 que j'avais rejetée originellement. En 1999-2000, il fallut soutenir sous la pression amicale voire affectueuse de mon second directeur de thèse et de ma femme ${ }^{9}$. Vingt ans de recherches et d'enseignement suffisaient mais la place de la Grande Guerre dans la relation bilatérale m'interpellait toujours. Ayant posé les bases de ce voisinage de 1898 à 1914, dans une étude finalement publiée en 2010-2011 ${ }^{10}$, dix ans après

\footnotetext{
5 J.-M. Delaunay, conférence sur l'histoire de la neutralité depuis les origines, Casa-Museo León y Castillo, avril 2014, Telde (Grande Canarie), dans un cycle universitaire dirigé par Javier Ponce Marrero.
}

${ }^{6}$ Le professeur Jean-Claude Allain (décédé en 2008) a succédé au professeur Duroselle, décédé en 1994. De 1979 à 1982, J.-M. Delaunay a été membre de la Section scientifique de la Casa de Velázquez (Cité Universitaire de Madrid).

7 Jean-Baptiste Duroselle me fit valoir en 1978 que de nombreux chercheurs travaillaient alors sur les relations franco-allemandes de la fin $X I X^{e}$-début $X X^{e}$ siècles (Bariéty, Poidevin, Guillen) ainsi que franco-britanniques (Andrew, Keiger) ou franco-italiennes (Milza), alors que personne ne se préoccupait des relations francoespagnoles de la période qui n'étaient plus aussi importantes à mes yeux que celles des $\mathrm{XVI}{ }^{\mathrm{e}}-\mathrm{XVIII}{ }^{\mathrm{e}}$ siècles...

${ }^{8} \mathrm{~A}$ l'exception de quelques travaux - de nature politico-migratoire - de Jean-René Aymes (période 1808-1848) et de Javier Rubio (période plus large), de la thèse d'Émile Témime sur les relations entre les deux pays de 1848 à 1868 - jamais publiée - du mémoire de maîtrise de François Héron, sur les relations durant la Première Guerre mondiale, et de quelques rares articles de revues publiés des deux côtés des Pyrénées.

9 J.-M. Delaunay, Méfiance cordiale. Les relations franco-espagnoles au début du XXe siècle (1899-1914), thèse d'État, 9 vol. dactylographiés, 3615 p., Université de Paris 1 Panthéon Sorbonne, 1999, direction Jean-Baptiste Duroselle (†) puis Jean-Claude Allain, soutenue le 29 janvier 2000 dans l'amphithéâtre Richelieu de la Sorbonne.

10 J.-M. Delaunay, Méfiance cordiale. Les relations franco-espagnoles au début du XXe siècle (1899-1914), 3 volumes, L'Harmattan, Paris 2011, 2800 p. 
la soutenance, je pouvais enfin avancer vers la guerre de 1914, mais j'avais déjà posé quelques jalons pour comprendre les liens entre une nation belligérante et une nation neutre.

Ainsi, j'avais participé très précocement à un numéro spécial de la défunte revue Historia 16, dès juillet 1981, par un bref article qui tendait à montrer que l'Espagne avait travaillé durant la Grande Guerre pour la victoire des alliés occidentaux, de par sa situation géographique, sa disponibilité migratoire autant que commerciale et sa disposition globalement amicale. Les intérêts étrangers, anglais, français et belges étaient alors dominants voire écrasants dans tous les secteurs de la vie économique du royaume ${ }^{11}$. En outre, une esquisse sur les relations franco-espagnoles de cette époque dramatique de I'histoire européenne avait été déjà ébauchée à travers un petit colloque à la Casa de Velázquez tenu en mai 1981, lors de mon séjour comme membre de la Section scientifique de cette institution française autour de laquelle s'était édifiée la Cité universitaire de Madrid dans les années 1920-1930. Présent avec divers collègues français (René Girault, Maurice Vaïsse, Émile Témime, Albert Broder, Paul Barrère, Germain Ayache), le professeur Duroselle avait proclamé que la neutralité espagnole allait de soi et ne pouvait être critiquée du point de vue national espagnol ce qu'approuvèrent les collègues espagnols présents (Manuel Espadas Burgos, Manuel Medina, Víctor Morales Lezcano, José Andrés Gallego, Gonzalo Sanz Casas, Miguel Alonso Baquer). Aucun intérêt majeur n'était menacé et rien ne pouvait justifier une entrée du royaume d'Alphonse XIII dans un conflit que tout le monde - on ne le dira jamais assez - croyait court. De ces échanges, pour des raisons désormais obscures, seul un compte-rendu a été tiré ${ }^{12}$. Enfin, j'avais encore pu me défaire de cette frustration originelle à travers une thèse de $3^{\mathrm{e}}$ cycle soutenue en 1988 sous la direction de J.-B. Duroselle - initialement pas très content de cet intermède de quelques années, cependant utile pour obtenir un poste universitaire - relative à l'histoire de la Casa de Velázquez et de ses antécédents sur le temps long (1909-1979) ${ }^{13}$. Entre 1914 et 1918, l'œuvre de propagande de l'Institut français de Madrid ancêtre de ladite Casa - accrochée à une politique culturelle d'avant-guerre déjà active avait été mise en valeur dans le cadre d'une relation neutre-belligérant qui $m$ 'avait alors frappé avant de me faire réfléchir à certains aspects de la vie diplomatique, autour de la information humanitaire et de la protection d'intérêts par puissance tierce. D'autres interventions - dans le cadre de colloques divers avaient aussi abordé la position de l'Espagne dans la Première Guerre mondiale. Nous y reviendrons.

Il n'est pas le lieu de revenir sur les causes de cette neutralité espagnole mais d'en esquisser les contours dans la relation bilatérale avec la France. Or c'est de la constance que jaillit la circonstance, de la permanence que vient la rupture.

\footnotetext{
${ }^{11}$ J.-M. Delaunay, "España trabajo para la victoria 1914-1918", Historia 16, Madrid, juillet 1981, p. 38-44.

12 J.-M. Delaunay,"Relations franco-espagnoles autour de la première guerre mondiale : colloque de Madrid (20-22 mai 1981)", Mélanges de la Casa de Velázquez, Madrid, 1982 (tome XVIII/2), p. 129-148.

${ }^{13} \mathrm{~J}$ J-M. Delaunay, Des Palais en Espagne. L'École des Hautes Études Hispaniques et la Casa de Velázquez au cœur des relations franco-espagnoles $d u X X^{e}$ siècle (1898-1979), thèse de $3^{e}$ cycle, 5 vol. dactylographiés, 5 vol., 1426 p., Université de Paris-1, 1987 (direction J.-B. Duroselle), soutenue le 30 janvier 1988 à la Sorbonne, publiée dans la Collection de la Bibliothèque de la Casa de Velázquez, n 10, Madrid, 1994, 670 p. (sur la Première guerre mondiale, p. 88-148).
} 


\section{DE LA CIRCONSTANCE}

En quoi cette nouvelle guerre générale européenne - un siècle après les luttes révolutionnaires et napoléoniennes - était-elle une rupture ? Bien sûr, par son poids colonial et technique, ce conflit devenait géographiquement - mais très inégalement - universel. Brisait-il pour autant les continuités imposées par une hostilité franco-prussienne devenue franco-allemande sous le regard vigilant d'un Royaume-Uni maître des sentinelles maritimes du continent, d'une Autriche-Hongrie vaste, mais disparate et enclavée, d'une Russie écartelée entre Orient et Occident, puissances au cœur et aux marches d'un espace enivré depuis des siècles par sa supériorité civilisatrice, coloniale, impériale ? On ne peut oublier que le plus grand personnage de l'histoire européenne, depuis la chute de Napoléon $\mathrm{I}^{\mathrm{er}}$, a été Otto von Bismarck. Personne n'arrive à la cheville de celui qui a forgé sur le dos de ses voisins, au cœur même de l'Europe, la grandeur bien réelle de l'Allemagne nouvelle ... jusqu'à nos jours. Toutes les autres puissances - France et Espagne parmi bien d'autres - se sont déterminées, définies par rapport à cette grande nation sans cesse ressuscitée depuis les temps dits barbares. La Grande Guerre n'est plus alors qu'une respiration internationale conjoncturelle de cette réalité hégémonique. Que pèsent alors les grands facteurs de la bilatéralité franco-espagnole tels que je les avais exposés lors d'une colloque à Béziers en 1993, entre géographie, démographie, sécurité, dialogue, exemplarité, prospérité, identité, séduction ${ }^{14}$. Ce sont ces éléments intangibles qu'il faudrait décliner sur le mode de l'affrontement général ou particulier : la guerre change toujours les atmosphères mais pas toujours les enjeux surtout entre un belligérant et un neutre dont les efforts ne se rejoignent que dans le retour à la paix, victorieuse pour l'un, équilibrée pour l'autre. Cela pose le statut de la neutralité des pays qui n'ont pas choisi d'entrer dans le conflit. Avec les États scandinaves, les Pays-Bas, la Suisse et le Saint-Siège, I'Espagne fut de ceux qui ont résisté inébranlablement à l'appel aux armes ${ }^{15}$. D'autres pays n'ont pu s'accrocher à cette neutralité. Ils entrèrent dans le conflit sous la pression des camps en lice - la fameuse course aux alliances périphériques - pour diverses raisons plus ou moins profondes : ainsi, de l'Empire ottoman et du Japon aux États-Unis en passant par la Bulgarie, la Roumanie, le Portugal, la Chine ou la Grèce ${ }^{16}$.

Il faut affirmer d'emblée que les circonstances de la guerre ont induit des phénomènes qui dépassent de loin le bilatéral strict. Avant tout, la frontière directe - géographique, souveraine - entre le(s) belligérant(s) et le(s) neutre(s) demeure intangible. La seule susceptible de changer se situe entre deux belligérants hostiles dont le vaincu devra céder le plus souvent du territoire au vainqueur. La France a bien connu cette mitoyenneté : en principe, la Belgique et le Luxembourg au nord, la Suisse et - plus étonnamment - l'Italie à l'est, l'Espagne au sud. L'Espagne quant à elle, forma initialement un bloc ibérique neutre. Mais le développement et le prolongement du conflit changent la donne : les deux premiers États cités sont envahis. L'Italie se proclama neutre avant d'entrer dans le conflit en 1915 mis

\footnotetext{
14 J.-M. Delaunay, "L'Espagne dans la politique générale française au XX $X^{\mathrm{e}}$ siècle", Images et influences de l'Espagne dans la France contemporaine, Ville de Béziers-Presses U. de Perpignan, 1994, p. 34-54 (colloque Béziers, 5 juin 1993).

15 J.-M. Delaunay,"L’Espagne devant la guerre mondiale, 1914-1919. Une neutralité profitable ?", Relations Internationales, $\mathrm{n}^{\circ}$ 160, 2015, p. 53-69 (colloque de Fribourg, Suisse, 24 mai 2014).

16 J.-M. Delaunay, "Les neutres européens [sauf Suisse]", Encyclopédie de la Grande Guerre 1914-1918, (dir. Stéphane Audoin-Rouzeau et Jean-Jacques Becker), Bayard, Paris, 2004, p. 855-866 [rééd. Édition du Centenaire, Bayard, Paris, 2013, p. 803-814].
} 
pas du côté initialement prévisible. Seules les frontières franco-espagnole et franco-suisse demeurèrent des limites entre pays à statut différent durant toute la guerre. La limite internationale pyrénéenne présente une autre particularité : la "bulle d'autonomie" d'Andorre qui fut apparemment incluse dans la sphère de neutralité espagnole.

De même, l'espionnage ou la recherche de renseignements en territoire neutre n'ont-t-il pas été franco-espagnols mais entre Alliés occidentaux et Empires centraux en territoire neutre. De même, la propagande auprès de l'opinion publique et des élites espagnoles fut plus audacieuse et plus rapide du côté des puissances germaniques - qui en avaient initialement davantage besoin - que du côté allié. Chez certains diplomates, ne la croyait-on pas inutile voire dangereuse et contre-productive auprès d'un public neutre que l'on supposait naturellement favorable à la cause du droit face à la force barbare du camp ennemi ? II s'agissait là d'enjeux multilatéraux où la victoire se forgeait aussi au front des neutres comme en témoignent des registres d'hôtel où l'on trouve les noms des agents de renseignements les plus divers ${ }^{17}$. Pourtant, il y eut des incidents franco-espagnols. Ainsi, en 1917, des agents français victimes de cercles germanophiles furent accusés - par les autorités du royaume de propagation d'idées supposées révolutionnaires à la suite des événements de Russie ${ }^{18}$.

Entre le multilatéral et le bilatéral strict, une zone mixte concerne davantage la sphère économique où la concurrence commerciale d'avant-guerre s'est longtemps maintenue jusqu'à la dernière année de la guerre (1918) quand les missions d'achat alliées/associées ont fini par s'imposer bien tardivement comme pour la coordination militaire - afin de limiter une hausse massive du prix des fournitures espagnoles. Ainsi, les besoins croissants en matières premières, en produits industriels ou agricoles furent-ils dus à l'effort de guerre et non aux besoins normaux des économies en temps de paix. Bref, de vastes secteurs d'activité ont été indéniablement bouleversés ou créés par l'éclatement de la guerre. Courte, les conséquences auraient été évidemment moins cruelles et moins coûteuses, mais la fixation des fronts fin 1914 allait donner aux neutres un poids croissant souvent déterminé par leur capacité d'action économique et leur liberté de mouvements commerciaux. On sait combien ces éléments ont été déterminants en vue d'assurer l'éclosion d'une bourgeoisie capitaliste - souvent rapidement anoblie - qui ne devait pas elle-même assumer le décollage d'un pays vite empêtré dans une guerre sporadique au sud du détroit de Gibraltar ${ }^{19}$.

Que l'Espagne neutre pouvait-elle apporter à la France qui s'était jetée à son corps défendant dans la mêlée ? Une rivalité allait immédiatement se développer entre les pays neutres dans une véritable course à la médiation et à la protection. Aspect largement ignoré des historiens, le royaume d'Alphonse XIII allait assurer la protection des intérêts français en Allemagne - une tâche lourde sous l'autorité de l'ambassadeur Polo de Bernabé - mais aussi en Palestine ottomane (le consul général Ballobar) et en Bulgarie, sans oublier l'aide apportée aux populations de Belgique et du nord occupé de

\footnotetext{
17 Ainsi au Palace Hotel de Madrid dont les registres de clientèle ont été étudiés par J.-M. Delaunay dans les années 1980. Allusion op. cit, note 16, p. 861 (éd. 2004), p. 809 (éd. 2013).

${ }^{18}$ Grosse documentation sur l'affaire des agents Chaumié, Gissot, Gilard (1917). Sur la diffusion des idées révolutionnaires, l'ouvrage de Francisco Romero Salvado, España 1914-1918. Entre la guerra y la revolución, Crítica, Barcelone, 1999.

19 L'ouvrage pionnier de Santiago Roldan, José García Delgado et Juan Muñoz, La formación de la sociedad capitalista en España 1914-1920, 2 vol., Cajas de Ahorro, Madrid, 1973.
} 
la France par l'infatigable ministre espagnol, Villalobar ${ }^{20}$. Elle assuma - devant la Suisse, qui l'eût cru ? de nombreuses autres missions de protection ${ }^{21}$. Cette action diplomatique ne doit pas être confondue, comme le font des auteurs courtisans, avec l'activité philanthropique à visée éminemment politique et diplomatique du souverain lui-même depuis le Palais royal, à travers un Bureau d'aide aux familles de prisonniers (Oficina pro cautivos). En renseignant ces proches des militaires des deux camps sur leurs prisonniers, leurs blessés ou leurs défunts - elle était destinée en réalité à assurer le succès d'une médiation royale qui permettrait l'organisation de la conférence de la Paix à Madrid! Sans résultats ${ }^{22}$. À la fin de la guerre, la Suisse, qui devait recevoir le siège de la future Société des Nations à Genève, fut bien la seule puissance neutre qui suscitera la sympathie des vainqueurs pour son énorme tâche humanitaire. Pas l'Espagne, dont le souverain sera vilipendé par Clemenceau et par d'autres pour sa duplicité même si on devait lui décerner la Médaille de la Reconnaissance française dès décembre 1918, puis, en mai 1920, la Médaille militaire ${ }^{23}$.

Sur un plan plus strictement bilatéral, la guerre engendra un affaissement de la présence humaine et économique française en Espagne, de par une mobilisation qui devait faire disparaître ou ralentir l'activité d'un grand nombre de petites ou moyennes entreprises. Des élites expatriées non mobilisées tentèrent de s'organiser à l'ombre d'une propagande improvisée, puis peu à peu animée sous la conduite originellement incertaine de leurs ambassades et de leurs consulats ${ }^{24}$. Dans le sens contraire, il faut en finir avec l'idée que ce serait la première guerre mondiale qui aurait accéléré voire initié l'immigration espagnole de masse dans les territoires français d'Europe et d'Afrique. Le mouvement était largement lancé avant le conflit, notamment au sud de la Méditerranée. La communauté espagnole était déjà la troisième en métropole au moment de l'éclatement du conflit, voire la seconde si l'on prend en compte le crochet migratoire vers l'Afrique du nord, encore plus ancien $^{25}$. Malgré un stop and go perceptible d'août à décembre 1914, le flux était reparti à la hausse,

20 J.-M. Delaunay, "L'Espagne, protectrice des intérêts français en Palestine au crépuscule de la domination ottomane, 1914-1918", Méditerranée, Moyen-Orient : deux siècles de relations internationales. Recherches en hommage à Jacques Thobie, L'Harmattan, Paris, 2003, p. 95-107, et du même, "En toute discrétion. L'Espagne, protectrice des intérêts français en Allemagne 1914-1919", Aux vents des Puissances. Hommages à Jean-Claude Allain, Presses de la Sorbonne Nouvelle, Paris, 2008, p. 195-208.

${ }^{21}$ J.-M. Delaunay, "L’Espagne, puissance protectrice, de la fin du XIX $X^{e}$ siècle au début du XXI ${ }^{e}$ siècle", Relations internationales, $n^{\circ} 143,2010$, p. 51-60 (avec introduction au colloque Entre guerres et ruptures, la protection dans les relations internationales, Genève, 28-29 mai 2010). Les fonds des ambassades d'Espagne à Paris et à Berlin sur cette question de la protection par puissance tierce sont aux archives du Palais royal et ne sont pas à confondre avec les fonds de l'œuvre particulière du roi.

22 J.-M. Delaunay, "Le "grand dessein européen" d'Alphonse XIII (1914-1918). De médiations en illusions", La présence des Bourbons en Europe XVI ${ }^{e}-X X l^{e}$ siècle, PUF, Paris, 2003, p. 321-335 (colloque Université Paris-IV Sorbonne, $1^{\text {er }}-2$ décembre 2000).

${ }^{23}$ Le nouvel ambassadeur désigné, Gabriel Alapetite, en décembre 1918, et le maréchal Joffre, en mai 1920, lui remirent les insignes et les diplômes au palais royal de Madrid.

${ }^{24}$ J.-M. Delaunay, "De l'incertaine existence d'élites hors de leur pays d'origine : le cas des Français en Espagne autour de la première guerre mondiale", Cahiers de l'Université de Pau et des Pays de l'Adour. Pyrenaica, Pau (1983), n $n^{\circ}$ 1, p. 132-136.

${ }^{25}$ J.-M. Delaunay, Méfiance cordiale, op. cit., vol. 1, p. 725-731, et vol. 2, p. 45-73. 
sous la protection d'un front stabilisé, d'un besoin urgent de main-d'œuvre par prolongement du conflit et d'un transfert d'activités industrielles vers le sud (Toulouse et Marseille notamment).

La population espagnole restée au pays, originellement peu au fait des enjeux et des évènements de la guerre, finit par se diviser grossièrement entre "aliadophiles» et germanophiles ${ }^{26}$, même s'il faut nuancer fortement les clivages entre les droites conservatrices supposées progermaniques et les gauches progressistes ou libérales pro-françaises ou pro-britanniques et enfin ne pas oublier les indifférents, les indécis ou les insouciants - les vrais neutres ? - qui n'avaient en général peur que d'une perspective : que leur pays soit entraîné dans la lutte. Les stéréotypes n'étaient pas absents des choix des habitants du royaume quand leur origine personnelle n'y était pas engagée. On reprochait à la France - $\mathrm{au}(\mathrm{x})$ Français ? - à des degrés très divers et en vrac, à la fois son intolérance anticléricale, sa présence économique et financière, son voisinage maritime (pêche), son arrogance impérialiste et culturelle, les figures de Louis XIV et de Napoléon I ${ }^{\mathrm{er}}$, son accueil des anarchistes et autres révolutionnaires, son attraction de main-d'œuvre et sa rivalité coloniale ${ }^{27}$. En tout cas, platoniquement ou pratiquement, la guerre pénétra fortement le tissu social espagnol même si certaines formes d'engagement ne concernèrent guère la plupart des habitants du royaume. Seuls, ceux qui devaient franchir la frontière française ou se lancer sur la mer se sentaient concernés. On leur demandait désormais des laissez-passer. Ce n'était pas toujours nécessaire. Ainsi, parmi les engagés espagnols dans l'armée française (Légion étrangère) - environ 1200 - l'immense majorité - non catalane - était déjà établie en France métropolitaine (72\%) ou en Afrique du nord (17\%) lors de l'éclatement du conflit $^{28}$. Après-guerre, sur le plan migratoire, le retour des Français au sud des Pyrénées fut plus mesuré. De même, des groupes religieux français réfugiés au sud des Pyrénées depuis la fin du siècle précédent et le début du suivant revinrent vers leur patrie d'origine dans le cadre de l'Union sacrée proclamée par le président de la République, Raymond Poincaré, en août 1914. Reliquats des luttes anticléricales d'avant-guerre, ces communautés disparurent ou repartirent finalement après le conflit ${ }^{29}$.

\footnotetext{
${ }^{26}$ L'ouvrage ancien mais pionnier de Fernando Diaz Plaja, Francófilos y germanófilos. Los españoles en la Guerra Europea, Dopesa, Barcelone, 1973. Paul Aubert et Eduardo González Calleja, dans Nidos de espías. España, Francia y la primera guerra mundial 1914-1918, Alianza, Madrid, 2014, ont fourni un gros travail mais ignorent la production la plus importante de J.-M. Delaunay ainsi Des Palais en Espagne, op. cit., et divers articles bien plus récents.
}

27 J.-M. Delaunay, "L'action diplomatique des pays belligérants et l'opinion publique espagnole durant la première guerre mondiale", Opinion publique et politique extérieure, tome II, 1915-1940, École Française de Rome, Rome, 1984, p. 229-234 (colloque École Française de Rome-Université de Milan, Rome, 16-20 février 1981), et du même, "1914. Les Espagnols et la guerre", Les sociétés européennes et la guerre de 1914-1918, Paris-Nanterre, 1990, p.117-132 (colloque Nanterre, Université Paris-X, 9-12 décembre 1988).

28 J.-M. Delaunay, "Tous Catalans ? Les combattants espagnols de l'armée française, 1914-1918", Des étoiles et des croix. Mélanges offerts à Guy Pedroncini, Economica, Paris, 1995, p. 309-323 (sur leurs lieux de résidence en 1914, p. 316). Les pourcentages sont une projection à partir de la seule lettre A. Dans l'art. cité, note 11, p. 40, Delaunay - alors tout jeune chercheur - ne récuse pas (à tort) le chiffre de 15000 volontaires espagnols dont une majorité de catalans, objet de manipulations actuelles liées à l'affirmation d'une nationalisme catalan indépendantiste. Encore du même auteur, les notices "Espagnols, Volontaires (1914-1918)" et "Légion étrangère espagnole", dans La Légion étrangère. Histoire et Dictionnaire, coll. Bouquins, Robert Laffont-Ministère de la Défense (dir. PaulAndré Comor), Paris, 2013, p. 345-347 et 539-541.

${ }^{29}$ J.-M. Delaunay, "La Grande Guerre ou la clé du retour", Mélanges de la Casa de Velázquez ${ }_{L}$ Madrid, 1983 (tome $\mathrm{XIX/1),} \mathrm{p.} \mathrm{347-368.} \mathrm{Ces} \mathrm{congrégations} \mathrm{étaient} \mathrm{venues} \mathrm{se} \mathrm{réfugier} \mathrm{en} \mathrm{Espagne} \mathrm{en} \mathrm{deux} \mathrm{vagues} \mathrm{(1880-1882} \mathrm{et} 1901$ 1914) évoquées dans les Mélanges de la Casa de Velázquez _ Madrid, 1981 (tome XVII), p. 291-319, et 1982 (tome 
Ce fut cependant pendant le conflit que devait disparaître la dette extérieure espagnole en mains françaises. De plus de $60 \%$ avant août 1914, elle devait glisser à environ $10 \%$ en 1920 . En outre, l'Espagne fut le $3^{\mathrm{e}}$ pays - et le premier neutre - à prêter à partir de 1916 de fortes sommes à la République combattante, loin il est vrai des États-Unis et du Royaume-Uni devenus les grands créanciers d'une France triomphante dans les larmes et les dettes. Des dépôts de titres espagnols en mains françaises (pignoración de valores), pour un montant estimé à plus de 330MF, furent engagés pour obtenir des crédits de fournitures exclusives de la part des banques de la péninsule. De cette situation inédite - l’Espagne créancière de la France - ne devaient sortir que tensions et rancœurs ${ }^{30}$.

\section{A LA CONSTANCE}

C'est pourtant la constance - la permanence, la continuité - qui l'emporte sur la circonstance - le changement, la rupture - dans l'évolution de la relation franco-espagnole autour de la Première Guerre mondiale. Tout d'abord, la victoire finale, d'un côté, la neutralité constante, de l'autre, ont permis aux régimes politiques des deux États de se maintenir en place sans troubles majeurs. Ce fut d'ailleurs le destin de tous les vainqueurs et de tous les neutres admis à adhérer à la nouvelle Société des Nations, une idée française d'avant-guerre (Léon Bourgeois) reprise avec succès par le président nord-américain (W. Wilson). Parmi les pays en guerre, presqu'aucun vaincu final n'a pu conserver ses institutions, Bulgarie exceptée. Les frontières européennes entre neutres et belligérants ne bougèrent pas à l'exception - qui confirme là encore la règle - du tracé germano-danois en 1920.

En Afrique, cependant, la défaite allemande entraîna la perte du Neu Kameroun obtenu par le Reich sur la France en 1911-1912 et replaça le Rio Muni espagnol - lieu de refuge d'une large partie des forces allemandes (1916) - en contact désormais exclusif avec la France le transformant totalement en enclave (colonie du Gabon au sud et à l'est, mandat du Cameroun au nord). Quant à la zone spéciale de Tanger, sur le détroit de Gibraltar, elle va demeurer une pomme de discorde entre les deux pays et le Royaume-Uni. Alphonse XIII avait brutalement fait miroiter la fin de la reconduction des crédits de fournitures accordés à la France si Tanger, incluse géographiquement dans la zone d'influence et de protectorat de son pays, ne devait pas revenir à l'Espagne comme prix de sa bienveillante attitude économique et humanitaire durant la guerre. L'accord de 1923, péniblement obtenu, calmera le jeu un temps ${ }^{31}$.

Sur la frontière - la couche la plus ancienne et la plus intangible des relations bilatérales - les chantiers des chemins de fer transpyrénéens et d'autres voies terrestres, parfois entrepris durant le conflit (pont du tramway Irun-Hendaye), purent reprendre. Certaines installations avaient servi de lieux de passage discrets, ainsi le tunnel de Canfranc sur la ligne Pau-Saragosse. Il fallut achever ces voies à l'avenir économique pourtant incertain comme l'avaient dit certains experts dès 1904-1905. Lancées à

XVIII/1), p. 259-287.

${ }^{30}$ A l'origine de cet endettement sur lequel nous reviendrons, J.-M. Delaunay, " Les crédits Urquijo et la France en guerre", Mélanges de la Casa de Velázquez, Madrid, 1984 (tome XX), p. 339-353. Les ouvrages de Lucien Petit et d'Henri Truchy sur les finances extérieures de la France en guerre sont indispensables, mais surtout la thèse d'État - non publiée - d'Albert Broder, Le rôle des intérêts économiques étrangers dans la croissance de l'Espagne au XIX siècle 1767-1924, U. Paris 1 Panthéon-Sorbonne (dir. P. Vilar), 1981, vol. 2, p. 633-680.

${ }^{31}$ Les mémoires inédites de l'ambassadeur de France à Madrid, Gabriel Alapetite (1919) - bientôt publiées par J.M. Delaunay - insistent beaucoup sur cette question. Nous y reviendrons dans Guerres d'Europe et d'Afrique. 
partir de 1908, deux lignes sur trois seront inaugurées en 1928-1929. La guerre fut ainsi une parenthèse qui vit cependant quelques tentatives de sabotage, notamment sur le tunnel ancien reliant Cerbère à Port-Bou sur la côte méditerranéenne.

Déjà perceptible avant la guerre, le mode de locomotion aérien fut littéralement bouleversé techniquement par la guerre, le militaire l'emportant naturellement sur le civil et le sportif jusqu'alors dominants. Mais le retour à la paix devait impulser la reconversion des appareils militaires en avions civils ou sportifs mais surtout de transport, permettant la création de lignes aériennes qui allaient animer la relation franco-espagnole de façon aventureuse puis régulière avec un prolongement vers I'Afrique occidentale puis l'Amérique du sud. Les lignes Latécoère, depuis Toulouse - où s'était réfugiée l'entreprise initiale - animèrent ainsi la zone-frontière européenne vers Barcelone et Alicante, mais aussi le Maroc, le Sahara occidental avant le grand saut transatlantique.

Sur le plan commercial, la reprise des négociations rendues nécessaires par le régime incertain du modus vivendi (1892) devait aboutir enfin à un traité en bonne et due forme en 1922, la guerre ayant joué là un rôle d'accélérateur des échanges, parfois pour des produits moins désirables qu'en temps de paix.

Bref, le conflit avait brisé parfois bien des élans d'avant-guerre mais ne put les faire disparaître. Ainsi là encore, l'immigration espagnole reprit dans les territoires français d'Europe et d'Afrique. II est vrai que la saignée de la masculinité française et le besoin de retour des femmes au foyer et à la maternité expliquent la reprise de ces mouvements d'arrivées massives d'hommes jeunes et valides qu'Espagnols, mais aussi Polonais, Italiens et coloniaux, allaient animer jusqu'à la grande crise des années $1930^{32}$.

Dans le domaine culturel, symbole d'une politique d'attraction, de séduction ou d'influence plus tard américanisée sous le vocable de "soft power" - la Grande Guerre n’a pas davantage bousculé les perspectives franco-espagnoles d'avant 1914. La lutte contre les rivaux germaniques - le "chantage au Teuton" - reprit de plus belle en Espagne mais il fallut y ajouter d'autres concurrents italiens (fascistes) et anglo-saxon, plus tardifs ${ }^{33}$. La poursuite d'un politique culturelle française active en Espagne fut donc à l'ordre du jour à la suite d'un renforcement des structures d'action du Quai d'Orsay qui sut attirer d'autres ministères et académies dans son projet d'influence. La guerre avait fait mûrir un projet de création d'une Casa de Velázquez déjà évoquée qui serait la sublimation des deux institutions distinctes que la France entretenait depuis longtemps à Rome, l'Académie de France (Ville Médicis) et l'École française (Palais Farnèse). Des instituts français furent établis à Saragosse (1919) et à Barcelone $(1922)^{34}$.

Outre-mer, la guerre fut une parenthèse là encore, comme elle le fut pour l'abandon de la mission du Rio Muni par les Pères spiritains français prévue en septembre 1914 au profit des Pères Clarétains espagnols, qui n'eut lieu que fin 1918, près de vingt ans après l'évacuation déjà évoquée des

\footnotetext{
32 Ainsi s'explique la loi de 1920 interdisant strictement l'avortement en France (jusqu'en 1975). De nombreux ouvrages traitent de l'immigration en France dans les années 1919-1939 (Bonnet, Schor, Ponty, etc...).

33 J.-M. Delaunay, "L'Espagne, un champ ouvert. Rivalités et illusions culturelles en péninsule ibérique (XIX-XX $\mathrm{e}$.)", Relations Internationales, Paris, 1987, n 50, p. 215-227.

34 J.-M. Delaunay, Des Palais en Espagne, op. cit., p. 147-182. À signaler que les archives de I'Institut français de Barcelone viennent d'être rapatriés aux AMAE-Nantes.
} 
garnisons françaises en 1901. Sur tout cela, nous reviendrons en détails. Si le voisinage en Afrique équatoriale ne fut pas trop conflictuel - nonobstant la plainte récurrente de forestiers français contre les autorités espagnoles, initiée à la fin $d u X X^{e}$ siècle et qui reprit force après la Grande Guerre I'absence d'occupation effective de la plus large partie de leur Sahara occidental par les forces espagnoles de Villa Cisneros valut encore bien des récriminations de la part des Français de Mauritanie qui eurent à souffrir de rezzous venus de ladite zone, rappelant les mêmes doléances depuis les années 1900. En 1920, les autorités de Madrid avaient pourtant fondé un poste à La Güera, en face de PortÉtienne, mais leur présence tardait à se manifester sur les groupes nomades de l'intérieur dans des zones que des méharistes français avaient pourtant sillonné en toute illégalité entre 1907 et 1913, notamment lors du fameux raid Mouret sur Smara (1913). La guerre n'avait pas changé grand chose là encore, même si de médiocres tentatives de pénétration d'agents allemands pour exciter les nomades avaient été déjouées. Les déboires des aviateurs français des lignes Latécoère - parfois otages des nomades en cas d'avaries - dans les années 1920 ne changeaient pas la donne d'avant-guerre. Les Espagnols tardaient à occuper leurs nouveaux espaces coloniaux.

Mais le grand dossier franco-espagnol sur la scène internationale fut incontestablement le Maroc. Toujours le Maroc. Un dossier d'avant-guerre. Depuis le début du siècle - et la première tentative d'entente de 1902 - les deux pays ne cessèrent de se mesurer l'un à l'autre pour obtenir l'espace le plus vaste possible ou la liberté la plus grande possible dans cette partie de l'Afrique encore insoumise. Les querelles n'avaient été que très péniblement réglées en 1912 mais le cas de Tanger demeurait en suspens. Une méfiance cordiale régnait encore après 1918. La fin de la guerre permit l'expulsion ou la neutralisation des agents allemands demeurés en zone espagnole autour de certains groupes indigènes aidés également par des déserteurs de la Légion étrangère.

Pourtant, ce fut la tendance déjà très ancienne d'une arrogance française envers l'Espagne qui se manifesta encore et toujours. L'orgueil de la victoire de 1918 - avec sa paix manquée de 1919 - ne devait pas améliorer la situation. On y reviendra. Ce sentiment - plus ou moins exprimé - de supériorité que les Espagnols reportaient à leur tour sur le Portugais et les Marocains qui considéraient les Africains noirs pour leurs capacités à être christianisés et utilisés sous forme de main-d'œuvre docile. Pourtant, pied de nez à l'Histoire, le sort de la France n'aurait pas été brillant si la victoire rapide du Reich en 1914 - qui n'était pas impossible ! - avait pu réduire le pays à un territoire ridicule blotti contre la frontière espagnole selon un document pangermaniste retrouvé dans les archives. En effet, en novembre 1914, en pleine élaboration de buts de guerre, une carte publiée par un Allemand Karl Miesnik, transmise par le ministre de France à La Haye, nous apprend que si la victoire des alliés dépècerait littéralement le Reich, la défaite serait également terrible pour eux. La France vaincue serait réduite sans pitié à un espace de taille médiocre, dans le sud-ouest, sur la frontière de l'Espagne, les départements des BassesPyrénées, des Hautes-Pyrénées, du Gers et le sud des Landes ainsi coincés entre l'immense empire allemand, au nord, et l'imposant royaume d'Espagne, au sud, devenus des géants. La France réduite en somme à une bulle semi-pyrénéenne, à peine plus grande qu'Andorre ${ }^{35}$ ! De même, une victoire des Empires centraux en Europe aurait pu modifier certaines limites en Afrique équatoriale ou ailleurs, mais

\footnotetext{
${ }^{35}$ AMAE-Paris La Courneuve 1914-1918/41, carte annexe à d. n²90, Légation-La Haye (Allizé) à MAE-Bordeaux (Delcassé), 20. 11. 1914, qui montre que le la Corse serait livrée à l'Italie finalement restée alliée des Empires centraux. Dans un souci d'équilibre (!), Miesnik avait également montré la nouvelle Europe, en cas de victoire alliée. La France se serait emparée de tout le sud et l'ouest de l'Allemagne, de l'Autriche, ainsi que de l'Italie du nord.
}

HISPANIA NOVA., 15 (2017), págs. 303-315

DOI. https://doi.org/10.20318/hn.2017.3491 
faute de victoire germanique, cette éventualité relève de la spéculation. On sera plus prudent et inquiet en octobre 1940 lors de l'entrevue de Hitler et de Franco à Hendaye ...

Finalement, la Grande Guerre n'a modifié la relation franco-espagnole que de façon périphérique. Les éléments fondamentaux de la relation demeurèrent semblables en 1918-1919 à ceux élaborés entre 1900 et 1914, dans une phase qui devait durer un demi-siècle environ, une relation africaine surdimensionnée au détriment d'une relation européenne toujours réelle - comment auraitelle pu cesser de l'être ? - mais partiellement négligée. Voisins toujours, rivaux longtemps. La Première Guerre mondiale avait involontairement montré la voie. S'il y a des neutralités qui tuent, il y a des guerres qui épuisent. II en sera de même durant la Seconde Guerre mondiale où, malgré sa défaite cuisante, la France de Pétain maintiendra ses possessions face à l'Espagne revendicatrice de Franco, pourtant épuisée ${ }^{36}$. Seule, la décolonisation générale va changer la donne profonde, dans les années 1950-1960, rejetant les deux pays vers leur destin européen commun.

Surtout, les deux pays vont être entrainés dans les années 1920 (jusque dans les années 1980) par de nouveaux courants plus idéologiques, totalitaires, essentiellement anti-capitalistes (socialismes, communismes, nationalismes, militarismes). Seule une politique européenne tempérée peut brider de nos jours ces forces agressives toujours sous-jacentes. Finalement, dans la relation franco-espagnole, la constance du voisinage a vaincu la violence de la circonstance. La première guerre mondiale fut une des premières expériences de sa mise à l'épreuve mais pas la plus difficile quand l'on songe au second conflit mondial inauguré, à travers des invasions extra-européennes (Ethiopie, Chine), par la guerre civile espagnole.

\footnotetext{
${ }^{36}$ La zone internationale de Tanger mise à part, au statut restauré en 1945.
} 